\title{
Pure feedback effects in absolute identification
}

\author{
SHUJI MORI \\ Nagasaki University, Nagasaki, Japan \\ and \\ LAWRENCE M. WARD \\ University of British Columbia, Vancouver, British Columbia, Canada
}

\begin{abstract}
To reveal the pure effects of trial-by-trial feedback on judgmental accuracy and sequential dependencies independent of global anchoring effects and other influences, we presented subjects with sequences consisting alternately (within an experimental session) of short runs of trials with feedback (feedback sequences) and without feedback (no-feedback sequences). In Experiments 1 and 2 (absolute identification of sound intensity and sound frequency, respectively), judgmental accuracy was the same in the feedback and the no-feedback sequences, contrary to previous results. Also, in the feedback sequences, the dependency of the current response on the immediately preceding stimulus was larger than that in the no-feedback sequences, while the dependency on the previous response was larger in the no-feedback sequences. In Experiment 3 (absolute identification of sound frequency), we attempted to separate the effects of the number of response categories on sequential dependencies from the effects of the number of stimuli. The results showed that the number of response categories had a larger effect than the number of stimuli on most aspects of performance, but that both affected sequential dependencies. These results are generally consistent with a theory of absolute identification in which feedback affects judgmental accuracy by improving long-term memory for judgmental anchors, while feedback affects sequential dependencies by altering response biases.
\end{abstract}

It is widely believed that the presence or absence of trial-by-trial feedback has a considerable effect on performance in the absolute identification task. Giving subjects feedback (correct response number for a presented stimulus) after each response has been found to improve judgmental accuracy (e.g., Braida \& Durlach, 1972; Chase, Bugnacki, Braida, \& Durlach, 1983; Eriksen, 1958; Mori, 1989: Siegel, 1972; Ward \& Lockhead, 1970) and to change the form of sequential dependencies of the current response on the immediately preceding stimulus and response (Mori, 1989; Ward \& Lockhead, 1970,1971 ). The main purpose of the present study is to deconfound the effects of feedback and those of judgmental accuracy on sequential dependencies by using a new experimental method, which we call the sequencealternating method (Mori, 1991), to hold accuracy constant. This allows the pure effects of feedback on sequential dependencies to be revealed and also has implications for the origin of feedback effects on accuracy. In the following sections, we first summarize pre-

This article is based on a doctoral dissertation by S.M., submitted to the University of British Columbia. The research was supported by a grant from the Natural Sciences and Engineering Research Council of Canada to L.M.W. We thank Odie Geiger, Richard Wright, Bill Turkel, and Steve Lindsay for assistance in conducting the experiments. Correspondence concerning this article should be addressed to $\mathrm{S}$. Mori, Department of Engineering, Toyama Prefectural University, 5180 Kurokawa, Kosugi-machi, Imizu-gun, Toyama 939-03, Japan (e-mail: mori@pu-toyama.ac.jp). vious studies on the effects of feedback, and then describe the present experiments.

\section{Effects of Feedback on Judgmental Accuracy}

Studies of absolute identification have often found improvement of judgmental accuracy by feedback, although the results vary from a difference of a few percent (Eriksen, 1958) to an improvement of more than $35 \%$ (Siegel, 1972). In fact, Eriksen (1958) reported that giving feedback sometimes actually decreased accuracy, measured by the amount of information transmission, in some conditions.

For sound intensity, Braida and Durlach and their colleagues (Braida \& Durlach, 1972; Chase et al., 1983; Purks, Callahan, Braida, \& Durlach, 1980) maintained that giving feedback improves intensity resolution. They measured intensity resolution by estimating $d^{\prime}$ values for adjacent pairs of stimuli, and found that feedback increased summed $d^{\prime}$ by about $20 \%-36 \%$ on the average. They also found that $d^{\prime}$ was higher at the extremes of the stimulus range than at the middle of the range (the resolution edge effect, discussed later).

From these and other results, Braida and Durlach (1972, 1988) proposed a model of sound-intensity resolution. The model is in the language of Thurstonian and signal detection theories (i.e., statistical decision theory) and is concerned exclusively with the ability to resolve sound intensities. In the model, it is assumed that one important process by which a subject identifies a particular sound intensity is by comparing the sensation arising from the sound with a context of similar remembered sensations 
from sounds experienced in the course of an experiment (the context coding mode). Specifically, the subject judges the sensation by estimating its position relative to remembered perceptual anchors, which, in most cases, are placed at the extremes of a given stimulus set. According to the model, the subject's judgment is limited by the precision of the memory for the context and the accuracy of mapping of the sound sensation to that context. In particular, the resolution edge effect reflects the operation of the perceptual anchors: estimates of the position of a sensation are more accurate the nearer it is to the perceptual anchors. To explain why feedback improved intensity resolution in their experiments, Purks et al. (1980) argued that a previous stimulus and its feedback serve to strengthen the perceptual anchors, and predicted that the resolution edge effect should be more pronounced when feedback is given. However, Chase et al. (1983) found that the improvement in sensitivity with feedback was roughly uniform for all the stimulus pairs in the experiment.

\section{Effects of Feedback on Sequential Dependencies}

Sequential dependencies of the current response $\left(\mathrm{R}_{n}\right)$ on the immediately preceding stimulus $\left(\mathrm{S}_{n-1}\right)$ and response $\left(\mathrm{R}_{n-1}\right)$ are invariably a characteristic of the absolute identification task (Garner, 1953; Holland \& Lockhead, 1968; McGill, 1957; Mori \& Ward, 1990; Ward \& Lockhead, 1970), and have important implications for models of the processes by which responses in this task are generated (Treisman, 1985; Ward \& Lockhead, 1971). Thus it is important to have a precise and consistent description of these dependencies and the variables that affect them across a broad range of experimental conditions.

Since feedback has been found to affect absolute identification accuracy, it is likely that it has important effects on the ubiquitous sequential dependencies as well. Yet only a few studies have examined the effects of giving trial-by-trial feedback on sequential dependencies. Ward and Lockhead $(1970,1971)$ reported that giving feedback increased the amount of information transmission, as observed in some other studies, and decreased the size of dependencies of the current response, $\mathrm{R}_{n}$, on both $\mathrm{S}_{n-1}$ and $\mathrm{R}_{n-1}$. In the absence of feedback, the dependency of $\mathrm{R}_{n}$ on $\mathrm{R}_{n-1}$ was much larger than that on $\mathrm{S}_{n-1}$, whereas in the presence of feedback, the dependency on $S_{n-1}$ was about the same as that on $R_{n-1}$.

Mori (1989) reported the effects of feedback in low- and high-information conditions. The amount of information available was manipulated by changing discriminabilities among stimuli, and the amount of information transmission was about 0.8 bits higher in the high-information condition. In the low-information condition, giving feedback had a negligible effect on the amount of information transmission, and it increased the dependency of $\mathrm{R}_{n}$ on $\mathrm{S}_{n-1}$ and decreased the dependency on $\mathrm{R}_{n-1}$. In the high-information condition, giving feedback increased the amount of information transmission by $16 \%$, and decreased the dependency of $\mathrm{R}_{n}$ on both $\mathrm{S}_{n-1}$ and $\mathrm{R}_{n-1}$.

Although these results do not provide a completely consistent picture of the effects of feedback on sequential dependencies, they seem to suggest that trial-by-trial feedback affects the sizes of the effects of $S_{n-1}$ and $R_{n-1}$ on $\mathrm{R}_{n}$ relative to one another. Models of the response process in absolute identification (Treisman, 1985; Ward \& Lockhead, 1971) agree that the effect of $\mathrm{R}_{n-1}$ should be relatively large in the absence of feedback-sometimes larger than the effect of $\mathrm{S}_{n-1}$-and that the presence of feedback should increase the size of the effect of $\mathrm{S}_{n-1}$ and decrease that of $\mathrm{R}_{n-1}$ relative to one another. This is consistent with some of the above results - that is, the dependency on $\mathrm{R}_{n-1}$ was larger than that on $\mathrm{S}_{n-1}$ in the absence of feedback (Ward \& Lockhead, 1970), while giving feedback increased the dependency on $\mathrm{S}_{n-1}$, and decreased the dependency on $R_{n-1}$ (Mori, 1989).

However, other results mentioned above did not show such differences in the effects of $\mathrm{S}_{n-1}$ and $\mathrm{R}_{n-1}$. We suggest that this is because the presence or absence of feedback also affected the degree to which stimuli and responses were positively correlated in those experiments (Treisman \& Williams, 1984; Ward \& Lockhead, 1971). As summarized earlier, feedback often improves judgmental accuracy, and higher accuracy implies a less variable relationship between stimuli and responses (characterized here as a higher positive correlation for reasons described in the section dealing with multivariate information analysis). A relatively high correlation between $\mathrm{S}_{n-1}$ and $\mathrm{R}_{n-1}$ would make the dependency of $\mathrm{R}_{n}$ on $\mathrm{R}_{n-1}$ similar to that on $\mathrm{S}_{n-1}$, as observed in the feedback experiment reported by Ward and Lockhead (1971). Also, Mori (1989) suggested that the effects of feedback on the subject's response process interacted with the amount of information available from stimuli in his experiment. It is likely that changes in the stimulusresponse correlation as a function of feedback in that experiment obscured the effects of feedback on sequential dependencies predicted by the models mentioned above.

In order to reveal the pure effects of feedback on sequential dependencies, uncontaminated by changes in stimulus-response correlation, that correlation must be held constant while feedback is varied. To do this, the present study used a sequence-alternating method, in which sequences of trials with feedback (feedback sequence) and those without feedback (no-feedback sequence) were alternated within an experimental session. We reasoned that the method would help the subjects reestablish their long-term memory of context (Braida \& Durlach, 1972, 1988) in each feedback sequence, so that in the following no-feedback sequence, the memory of context would remain as accurate as in the feedback sequence. We thus predicted that accuracy and the stimulusresponse correlation would be unaffected by the presence of feedback. We used about 30 trials in each sequence, because Eriksen (1958) found that accuracy re- 
mained constant for at least 26 trials without feedback after the same experiment with feedback. ${ }^{1}$

\section{EXPERIMENT 1}

The first purpose of this experiment was to test the prediction that the sequence-alternating method would be effective in keeping judgmental accuracy constant in the feedback and no-feedback sequences. For that purpose, the stimuli to be judged in this experiment were sound-intensity levels identical to those used in some of Braida and Durlach's (1972) experiments, in which they found a $20 \%$ difference between summed $d^{\prime}$ with feedback and that without feedback. If our prediction was correct, in our experiment judgmental accuracy (or sensitivity) would be the same in the feedback and no-feedback sequences.

The second purpose of this experiment was to confirm that under our conditions, the effects of feedback on sequential dependencies would differ as a function of information available to the subject. For this purpose, we manipulated information by changing the range of soundintensity levels used as stimuli. Previous studies (Braida \& Durlach, 1972; Luce, Green, \& Weber, 1976) have shown that both intensity resolution and information transmission increase with the range of sound intensities to be judged.

\section{Method}

\section{Subjects}

Six students of the University of British Columbia, one of them the first author, participated in this experiment. Four were male and 2 were female, and none had any difficulty hearing the tones used as stimuli.

\begin{abstract}
Apparatus and Stimuli
A Hewlett-Packard Vectra ES/12 computer system controlled the presentation of the stimuli and recorded the responses, which were made on a dimly red-illuminated, standard computer-type keyboard. The tones were delivered diotically through Koss Pro4AAA earphones and amplified and gated by an elect ronic switch (2.5-msec rise and fall times). The stimuli were 10 intensity levels of a $1000-\mathrm{Hz}, 500-\mathrm{msec}$ pure tone. There were two sets of stimuli. In the large-range set, the intensity levels ranged from 50 to $86 \mathrm{~dB}$ in $4-\mathrm{dB}$ steps. In the small-range set, the intensity levels ranged from 77 to $86 \mathrm{~dB}$ in $1-\mathrm{dB}$ steps. The sets of tones were identical to those used in Braida and Durlach's (1972) Experiments 2, 4, and 6.
\end{abstract}

\section{Procedure}

Feedback sequences and no-feedback sequences were alternated within a session. The feedback sequence was always given first, followed by the no-feedback sequence. There were five alternations, and 30 trials in one sequence. Thus, one session consisted of 300 trials, half in feedback sequences and the other half in no-feedback sequences. The subjects were instructed to identify the 10 stimuli and to make responses in a consistent way in both the feedback and the no-feedback sequences. They were presented with the 10 stimuli and the required responses before they started the identification trials. Trials were self-paced. There were four sessions for each of the large-and small-range sets of tones, and each subject made 60 judgments for each tone of each set of stimuli in both the feedback and the no-feedback sequences. The order of the large- and small- range conditions was counterbalanced across subjects. Each subject did a 30 -min practice session before each session.

\section{Data Analysis}

Performance in this experiment was analyzed by a sensitivity measure $\left(d^{\prime}\right)$, multivariate information analysis, and multiple regression analysis.

Sensitivity measure. Following Lippmann, Braida, and Durlach (1976), we obtained maximum-likelihood estimates of a measure of sensitivity, $d_{i, i+1}^{\prime}$, to each adjacent pair of stimuli $\left(\mathrm{S}_{i}\right.$ and $\left.\mathrm{S}_{i+1}\right)$, as follows. ${ }^{2}$ First, the stimulus-response confusion matrices were pooled across subjects in order to obtain enough data to calculate stable estimates of $d_{i, i+1}^{\prime}$. Second, the normal deviate $Z_{i}(j)$ was calculated for all $\operatorname{Pr}(\mathrm{R} \geq j \mid \mathrm{S}=i)$-the probability of response to Stimulus $i$ being $j$ or larger - of the pooled confusion matrix, and $d_{i, i+1}^{\prime}$ was obtained by averaging the difference $\left[Z_{i}(j)-Z_{i+1}(j)\right]$ over all $j$ for which both $\operatorname{Pr}(\mathrm{R} \geq j \mid \mathrm{S}=i)$ and $\operatorname{Pr}(\mathrm{R} \geq j \mid \mathrm{S}=i+1)$ were between .01 and .99 . Using these as initial estimates, the maximumlikelihood estimates of $d_{i, i+1}^{\prime}$ were obtained by the Newton-Raphson iteration method for solving simultaneous nonlinear equations. Since such an estimate of $d^{\prime}$ is additive (Braida \& Durlach, 1972, 1988; Lippmann et al., 1976), we summed all $d_{i, i+1}^{\prime}$ values for the set of stimuli used in the experiment. Summed $d^{\prime}$ (total sensitivity in Braida and Durlach's term) is a useful index of intensity resolution (Braida \& Durlach, 1972, 1988) and can also be used to measure stimulus resolution in other modalities (e.g., sound frequency). Note that since all of the data available are used to arrive at a single number for each condition, the usual statistical tests cannot be applied to the summed $d^{\prime}$. However, such numbers are quite stable and even replicable across experiments for particular conditions (Braida \& Durlach, 1972). In lieu of statistics, we relied on consistent patterns of results for valid conclusions for this measure.

Multivariate information analysis. Multivariate information analysis is an extension of the usual one-input one-output information analysis (i.e., information transmission from input to output) to analyze relations among more than two variables in categorical data (Garner, 1962; McGill, 1954). To analyze sequential dependencies on $S_{n-1}$ and $R_{n-1}$, we used the following equation for multivariate information analysis:

$$
\begin{aligned}
\mathrm{U}\left(\mathrm{R}_{n}\right)= & \mathrm{U}\left(\mathrm{R}_{n}: \mathrm{S}_{n}\right)+\mathrm{U}\left(\mathrm{R}_{n}: \mathrm{S}_{n-1} \mid \mathrm{S}_{n}\right)+\mathrm{U}\left(\mathrm{R}_{n}: \mathrm{R}_{n-1} \mid \mathrm{S}_{n}, \mathrm{~S}_{n-1}\right) \\
& +\mathrm{U}\left(\mathrm{R}_{n} \mid \mathrm{S}_{n}, \mathrm{~S}_{n-1}, \mathrm{R}_{n-1}\right)
\end{aligned}
$$

$\mathrm{U}\left(\mathrm{R}_{n}\right)$ is response information, $\mathrm{U}\left(\mathrm{R}_{n}: \mathrm{S}_{n}\right)$ is information transmission from stimulus $\left(S_{n}\right)$ to response $\left(R_{n}\right), U\left(R_{n}: S_{n-1} / S_{n}\right)$ is information transmission from $S_{n-1}$ to $R_{n}$ with the effect of $S_{n}$ eliminated, and $U\left(R_{n}: R_{n-1} \mid S_{n}, S_{n-1}\right)$ is information transmission from $R_{n-1}$ to $R_{n}$ with the effects of $S_{n}$ and $S_{n-1}$ excluded. $U\left(R_{n} \mid S_{n}, S_{n-1}, R_{n-1}\right)$ is the residual amount of response information after the effects of $\mathrm{S}_{n}, \mathrm{~S}_{n-1}, \mathrm{R}_{n-1}$ are eliminated. $\mathrm{U}\left(\mathrm{R}_{n}: \mathrm{S}_{n-1} \mid \mathrm{S}_{n}\right)$ and $\mathrm{U}\left(\mathrm{R}_{n}: \mathrm{R}_{n-1} \mid \mathrm{S}_{n}, \mathrm{~S}_{n-1}\right)$ are used as a measure of the sequential dependency on $S_{n-1}$ and of the sequential dependency on $R_{n-1}$, respectively. See the Appendix for the detailed calculation of each term.

The great advantage of using multivariate information analysis is that statistically it is a nonmetric technique that is applicable to categorical data, such as those obtained from an absolute identification experiment (Krippendorff, 1986). However, recent studies (Houtsma, 1983; Mori, 1991) have found that such information measures are inflated for a small number of observations relative to the number of variables in the calculation. Although the results of computer simulations and empirical experiments (Mori, 1991) have shown that the estimates of information measures eventually reach an asymptotic level as the number of observations is increased, this would require a very large number of observations (sometimes more than 900 observations per stimulus).

In the present study, we used a method proposed by Mori (1991) to correct the overestimation of information measures in Equa- 
tion 1. First, the data of each subject were pooled as different combinations of individual data, and the information measures in Equation 1 were calculated from the pooled data. For example, there were six subjects in this experiment, and the number of combinations selecting $r$ subjects from 6 is calculated by ${ }_{6} \mathrm{C}_{r}$, where $r=$ $1-6$. When $r=1$, the information measures were calculated from each individual's data and then averaged. When $r=2$, there are ${ }_{6} C_{2}=15$ possible combinations and the information measures were calculated from the pooled data of each combination and averaged. When $r=6,{ }_{6} \mathrm{C}_{6}=1$ and all individual data were pooled and the information measures were calculated from them. Second, the resulting information measures were plotted against the number of pooled observations per stimulus (from 60 to 360 observations). Third, the same information measures were calculated from data produced by computer simulations ${ }^{3}$ (Mori, 1991) as a function of the number of observations per stimulus (from 10 to 900 observations), and fitted to those of the pooled data. For each pooled-data set, the fitted computer simulations were chosen in such a way that a single set of parameters could generate the information measures that fit best (by eye) those from the pooled data simultaneously. In general, the fits were reasonably good [except for $\mathrm{U}\left(\mathrm{R}_{n}: \mathrm{R}_{n-1}\right.$ $\left.\mathrm{S}_{n}, \mathrm{~S}_{n-1}\right)$ ], and the fitted information measures from the computer simulation data reached asymptotic levels as the number of observations increased. Finally, these asymptotic levels were taken as unbiased estimates of those from the pooled data.

The computer simulations and the correction method (and its advantages over other correction methods) are discussed extensively in Mori (1991). However, two points should be mentioned here. First, the correction method uses all of the subjects' data in an experiment to make a single estimate of every information measure in Equation 1, so that traditional statistical analyses cannot be applied to the information measures. However, the results of the computer simulations have shown that estimates of those information measures are very stable, with standard deviations of $0.010-0.015$ bits for 10 repetitions, especially when the number of observations per stimulus is greater than 120 . Therefore, we assume that the (unbiased) estimates of information measures obtained by the correction method are stable, within a range of $0.010-0.015$ bits, in the present study. In graphs of the information measures, we placed error bars of 0.015 bits to aid in determining significant differences among them.

Second, we failed to obtain reasonable fits to the estimates of $\mathrm{U}\left(\mathrm{R}_{n}: \mathrm{R}_{n-1} \mid \mathrm{S}_{n}, \mathrm{~S}_{n-1}\right)$ in the present study, because they showed a pattern quite different to those of the other measures (Mori, 1991). As an alternative, we calculated the estimate of $\mathrm{U}\left(\mathrm{R}_{n}: \mathrm{R}_{n-1} \mid \mathrm{S}_{n}, \mathrm{~S}_{n-1}\right)$ from the unbiased estimates of other information measures by using the following equation:

$$
\begin{aligned}
\mathrm{U}\left(\mathrm{R}_{n}: \mathrm{R}_{n-1} \mid \mathrm{S}_{n}, \mathrm{~S}_{n-1}\right)= & \mathrm{U}\left(\mathrm{R}_{n}\right)-\mathrm{U}\left(\mathrm{R}_{n}: \mathrm{S}_{n}\right)-\mathrm{U}\left(\mathrm{R}_{n}: \mathrm{S}_{n-1} \mid \mathrm{S}_{n}\right) \\
& -\mathrm{U}\left(\mathrm{R}_{n} \mid \mathrm{S}_{n}, \mathrm{~S}_{n-1}, \mathrm{R}_{n-1}\right)
\end{aligned}
$$

The value of $\mathrm{U}\left(\mathrm{R}_{n}: \mathrm{R}_{n-1} \mid \mathrm{S}_{n}, \mathrm{~S}_{n-1}\right)$ calculated from Equation 2 is thus an estimate from other estimates, and it may not be as accurate an estimate as would be obtained from a reasonable fit of simulation results. However, estimates from Equation 2 should be better than uncorrected estimates or those from an unsatisfactory simulation fit.

Multiple regression analysis. Multiple regression analysis is frequently used to analyze sequential dependencies in scaling tasks (e.g., Green, Luce, \& Duncan, 1977; Jesteadt, Luce, \& Green, 1977; Schifferstein \& Frijters, 1992; Ward, 1979, 1982, 1985, 1986, 1987). Following previous studies, we used two types of analysis in the present study. One type of analysis was an ordinal multiple regression of the form

$$
\mathrm{R}_{n}=\alpha_{0}+\alpha_{1} \mathrm{~S}_{n}+\alpha_{2} \mathrm{~S}_{n-1}+\alpha_{3} \mathrm{R}_{n-1}+\epsilon_{n},
$$

where $\alpha_{0}$ is the intercept, $\alpha_{1}, \alpha_{2}$, and $\alpha_{3}$ are regression coefficients, and $\epsilon_{n}$ is the error term. $\mathrm{R}^{2}$ increases and regression coeffi- cients were derived by hierarchical multiple regression, where $\mathrm{S}_{n}$, $S_{n-1}, R_{n-1}$ were entered in that order. The other type was a timeseries regression of the form

$$
\mathrm{R}_{n}=\beta_{0}+\beta_{1} \mathrm{~S}_{n}+\beta_{2} \mathrm{~S}_{n-1}+\rho \epsilon_{n-1}+\mathrm{u}_{n}
$$

(DeCarlo, 1992; DeCarlo \& Cross, 1990), where $\rho \epsilon_{n-1}+u_{n}$ is a first-order autoregressive error process with $\epsilon_{n-1}$ representing systematic error and $\mathrm{u}_{n}$ random error. DeCarlo and Cross (1990) and DeCarlo (1992) interpreted the autoregressive parameter $\rho$ as indicating the effect of frame of reference on scaling responses. We take it as a measure of dependency on $R_{n-1}$.

Two remarks should be made about the above application of multiple regression analysis. First, although regression models assume metric properties in data, most analyses can be performed on categorical variables to test whether the regression coefficients are positive or negative, or significantly different from zero (McNicol, 1980). The stimuli in this experiment (and those in the other experiments of the present study) were ordered along continuous dimensions, and the set of responses corresponded to the order of stimuli. Therefore, it may be justified to apply multiple regression analysis to data from the present experiments (also see Mori \& Ward, 1990).

Second, Luce, Green, and their colleagues (e.g., Green et al., 1977; Jesteadt et al., 1977) have shown in scaling tasks that the regression coefficient of $\mathrm{R}_{n-1}$ depends on the difference between $\mathrm{S}_{n}$ and $\mathrm{S}_{n-1}$. This is also demonst rated by computing the correlation between $R_{n}$ and $R_{n-1}$ as a function of the difference between $S_{n}$ and $\mathrm{S}_{n-1}$. The correlation coefficients are usually high when $\mathrm{S}_{n}$ is close to $\mathrm{S}_{n-1}$, but they drop to near or below zero when $\mathrm{S}_{n}$ and $\mathrm{S}_{n-1}$ are far apart. It is possible that a similar second-order dependency appears in absolute identification data and biases measures of sequential dependencies, especially those of $\mathrm{R}_{n}$ on $\mathrm{R}_{n-1}$, that ignore them. In fact, such second-order dependencies do appear in absolute identification data, but they are much smaller than those found in scaling data (Mori, 1994). Indeed, they are small enough in conditions such as those used here that they may safely be ignored for our purposes (Mori, 1994).

\section{Results}

\section{Sensitivity Measure}

Figure 1 presents $d_{i, i+1}^{\prime}$ for each of the 9 adjacent pairs of stimuli in the feedback and the no-feedback sequences, for the small- and large-range conditions. Values of $d^{\prime}$ were generally higher in the large-range condition than in the small-range condition. In the small-range condition, $d^{\prime}$ values showed no consistent change with stimulus intensity levels. In the large-range condition, $d^{\prime}$ values were higher at the edge of the stimulus set, particularly at the high-intensity edge. These results are consistent with those of Braida and Durlach (1972) and Chase et al. (1983).

As is readily seen in Figure 1, there was no systematic difference in $d^{\prime}$ between the feedback and no-feedback sequences in the small-range condition. In the largerange condition, $d^{\prime}$ values at the edges of the stimulus range were slightly higher in the no-feedback sequences than in the feedback sequences. These observations are supported by the total sensitivities ( $d^{\prime}$ summed across adjacent pairs of stimulus intensities), which are 5.0 (feedback) and 4.9 (no-feedback) in the small-range condition, and 8.9 for both sequences in the large-range condition. These values are also comparable to those of Braida and Durlach's (1972) Experiments 2, 4, and 6, which used the same stimuli as ours. They obtained total 

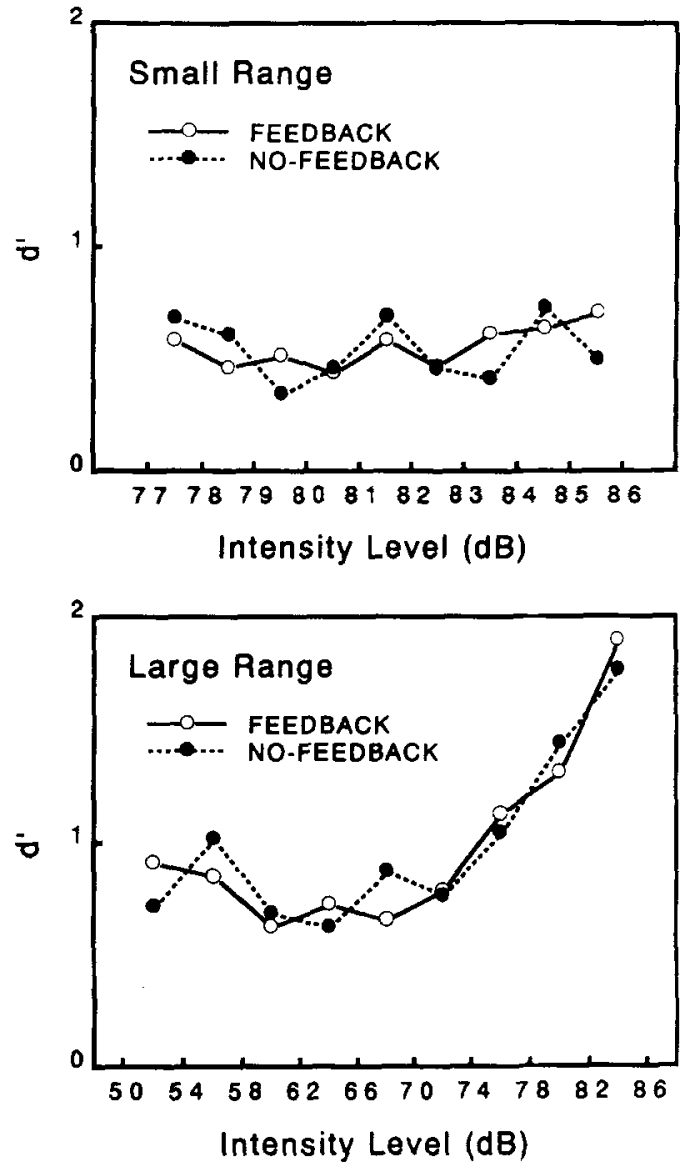

Figure 1. Sensitivity measures ( $d^{\prime}$ values) to adjacent pairs of stimuli in Experiment 1.

sensitivities of about 5.8 (with feedback) and 5 (without feedback) for the small range, and of 10.1 (with feedback) and 8.0 (without feedback) for the large range. However, the roughly $20 \%$ improvement they observed when feedback was given was not observed under our conditions.

\section{Multivariate Information Analysis}

Table 1 presents the results of the multivariate information analysis. The estimates of $\mathrm{U}\left(\mathrm{R}_{n}: \mathrm{S}_{n}\right)$ (information transmission) showed a tendency very similar to that of $d^{\prime} . \mathrm{U}\left(\mathrm{R}_{n}: \mathrm{S}_{n}\right)$ was higher by about 0.55 bits in the large-

Table 1

Results of Multivariate Information Analysis: Experiment 1

\begin{tabular}{lcccc}
\hline Sequence & $\mathrm{U}\left(\mathrm{R}_{n}\right)$ & $\mathrm{U}\left(\mathrm{R}_{n}: \mathrm{S}_{n}\right)$ & $\mathrm{U}\left(\mathrm{R}_{n}: \mathrm{S}_{n-1} \mid \mathrm{S}_{n}\right)$ & $\mathrm{U}\left(\mathrm{R}_{n}: \mathrm{R}_{n-1} \mid \mathrm{S}_{n}, \mathrm{~S}_{n-1}\right)$ \\
& \multicolumn{4}{c}{ Small Range } \\
Feedback & 3.316 & .887 & .179 & .423 \\
No-feedback & 3.320 & .858 & .116 & .495 \\
& \multicolumn{5}{c}{ Large Range } \\
Feedback & 3.303 & 1.424 & .142 & .222 \\
No-feedback & 3.294 & 1.422 & .098 & .301 \\
\hline
\end{tabular}

range condition than in the small-range condition. For both range conditions, there was little difference between $\mathrm{U}\left(\mathrm{R}_{n}: \mathrm{S}_{n}\right)$ of the feedback sequences and that of the no-feedback sequences.

As observed in previous studies (e.g., Mori, 1989, $1991), \mathrm{U}\left(\mathrm{R}_{n}: \mathrm{S}_{n-1} \mid \mathrm{S}_{n}\right)$ and $\mathrm{U}\left(\mathrm{R}_{n}: \mathrm{R}_{n-1} \mid \mathrm{S}_{n}, \mathrm{~S}_{n-1}\right)$, the measures of sequential dependencies on $S_{n-1}$ and $R_{n-1}$, showed an inverse relation with $\mathrm{U}\left(\mathrm{R}_{n}: \mathrm{S}_{n}\right)$; they were both larger in the small-range condition than in the largerange condition. In both conditions, $\mathrm{U}\left(\mathrm{R}_{n}: \mathrm{R}_{n-1} \mid \mathrm{S}_{n}, \mathrm{~S}_{n-1}\right)$ was larger than $U\left(R_{n}: S_{n-1} \mid S_{n}\right)$, and they showed a pattern opposite to each other. While $U\left(R_{n}: S_{n-1} \mid S_{n}\right)$ was larger in the feedback sequences than in the no-feedback sequences, $\mathrm{U}\left(\mathrm{R}_{n}: \mathrm{R}_{n-1} \mid \mathrm{S}_{n}, \mathrm{~S}_{n-1}\right)$ was larger in the nofeedback sequences than in the feedback sequences.

\section{Multiple Regression Analysis}

Tables 2 and 3 present the results of the multiple regression analyses. Table 2 presents average $R^{2}$ increases obtained for Equation 3. $\mathrm{S}_{n}$ added most to $\mathrm{R}^{2}$, and the $\mathrm{R}^{2}$ increases were larger in the large-range condition than in the small-range condition. Consistent with $d^{\prime}$ and $\mathrm{U}\left(\mathrm{R}_{n}: \mathrm{S}_{n}\right)$, they were almost identical in the feedback and the nofeedback sequences. However, the $R^{2}$ increases by $S_{n-1}$ and $R_{n-1}$ in the feedback sequences showed a pattern opposite to those in the no-feedback sequences. In the feedback sequences, $\mathrm{S}_{n-1}$ increased $\mathrm{R}^{2}$ more than $\mathrm{R}_{n-1}$ did, but in the no-feedback sequences, $R_{n-1}$ increased $R^{2}$ more than $\mathrm{S}_{n-1}$ did.

Table 3 presents the results for the two types of multiple regression analysis (Equations 3 and 4 ). The coefficients of $S_{n}$ and $\mathrm{R}^{2}$ were almost identical in both analyses. The coefficients of $\mathrm{S}_{n}\left(\alpha_{1}\right.$ and $\left.\beta_{1}\right)$ were larger in the large-range condition than in the small-range condition. In the small-range condition, the coefficients were almost identical in the feedback and no-feedback sequences. In the large-range condition, they were slightly larger in the no-feedback sequences than in the feedback sequences. However, the two types of analysis yielded somewhat different patterns for the sequential dependencies on $S_{n-1}$ and $R_{n-1}$. The major difference was in the coefficients of $S_{n-1}$. The coefficients obtained for Equation $3\left(\alpha_{2}\right)$ were positive in the feedback sequences and negative in the no-feedback sequences, while those obtained for Equation $4\left(\beta_{2}\right)$ were positive in both the feedback and the no-feedback sequences. A similar difference between the coefficients of $S_{n-1}$ for the two types of analysis has also been observed in studies of psychophysical scaling judgments (DeCarlo \& Cross, 1990). The coefficients of $\mathrm{R}_{n-1}$ for Equation $3\left(\alpha_{3}\right)$ and autoregressive parameters for Equation $4(\rho)$ were very similar to each other; they were positive in both feedback and no-feedback sequences and much larger in the nofeedback sequences than in the feedback sequences. With the exception of $\alpha_{2}$ for Equation 3, the coefficients of $S_{n-1}$ and $R_{n-1}$ were larger in the small-range condition than in the large-range condition, consistent with the results of the multivariate information analysis (Table 1 ). 
Table 2

Average $\mathbf{R}^{2}$ Increase in Equation 3

\begin{tabular}{|c|c|c|c|c|c|c|}
\hline & \multicolumn{6}{|c|}{ Sequence } \\
\hline & \multicolumn{3}{|c|}{ Feedback } & \multicolumn{3}{|c|}{ No-Feedback } \\
\hline & $\mathrm{S}_{n}$ & $S_{n-1}$ & $\mathrm{R}_{n-1}$ & $\mathrm{~S}_{n}$ & $S_{n-1}$ & $\mathbf{R}_{n-1}$ \\
\hline \multicolumn{7}{|c|}{ Experiment 1} \\
\hline \multicolumn{7}{|l|}{ Range } \\
\hline Small & $.722^{\dagger}$ & $.015^{+}$ & .003 & $.715^{\dagger}$ & $.004^{*}$ & $.028^{\dagger}$ \\
\hline Large & $.862^{\dagger}$ & $.004^{*}$ & .000 & $.872^{\dagger}$ & $.002^{*}$ & $.007^{\dagger}$ \\
\hline \multicolumn{7}{|c|}{ Experiment 2} \\
\hline \multicolumn{7}{|c|}{ Number of Stimuli } \\
\hline 6 & $.962^{\dagger}$ & .001 & .000 & $.954^{\dagger}$ & .001 & .001 \\
\hline 11 & $.930^{\dagger}$ & .005 & .000 & $.908^{\dagger}$ & .000 & $.004^{*}$ \\
\hline 16 & $.948^{\dagger}$ & .001 & .000 & $.939^{\dagger}$ & $.001^{*}$ & $.002^{\dagger}$ \\
\hline
\end{tabular}

\section{Discussion}

The results of this experiment confirmed our prediction that the sequence-alternating method would be effective in keeping the subjects' judgmental accuracy (or sensitivity) roughly constant in the feedback and nofeedback sequences. The $d^{\prime}$ and $\mathrm{U}\left(\mathrm{R}_{n}: \mathrm{S}_{n}\right)$ measures showed that the subjects' judgment accuracy (or sensitivity) was almost identical in the feedback and nofeedback sequences.

The manipulation of stimulus information by changing the stimulus range was also successful in this experiment. $\mathrm{U}\left(\mathrm{R}_{n}: \mathrm{S}_{n}\right)$ was larger in the large-range condition than in the small-range condition, and the other measures of the effect of $\mathrm{S}_{n}$ on $\mathrm{R}_{n}$ (i.e., $d^{\prime}, \mathrm{R}^{2}$ increase) showed the same results. In addition to this range effect, $d^{\prime}$ values were larger at the edges of the stimulus range (resolution edge effect) in the large-range condition, as observed by Braida and Durlach (1972). The measures of sequential dependencies on $S_{n-1}$ and $R_{n-1}$ were generally larger in the small-range condition than in the large-range condition, consistent with theories that predict an inverse relation between the amount of $U\left(\mathrm{R}_{n}: \mathrm{S}_{n}\right)$ and the magnitude of sequential dependencies (e.g., Mori, 1989, 1991).

The manipulation of amount of information available did not affect the effects of feedback on the sequential dependencies. The measures of the dependency on $S_{n-1}$ were generally larger in the feedback sequences than in the no-feedback sequences (with the exception of $\alpha_{2}$ obtained for Equation 3), while those of the dependency on $R_{n-1}$ were larger in the no-feedback sequences than in the feedback sequences. In addition, some of the analyses $\left(\mathrm{R}^{2}\right.$ increases and Equation 4) indicated that in the feedback sequences, the dependency on $\mathrm{S}_{n-1}$ was larger than that on $\mathrm{R}_{n-1}$, while the pattern was the opposite in the no-feedback sequences. Since $\mathrm{U}\left(\mathrm{R}_{n}: \mathrm{S}_{n}\right)$ was the same in the feedback and no-feedback sequences, the differences between the sequential dependencies of the two sequences reflect the pure feedback effects on sequential dependencies, uncontaminated by differences in the stimulus-response correlation.

\section{EXPERIMENT 2}

One purpose of the second experiment was to examine the generality of the results of Experiment 1 . In Experiment 1 , although the accuracy was kept at the same level in the feedback and no-feedback sequences, it was rather poor: $\mathrm{U}\left(\mathrm{R}_{n}: \mathrm{S}_{n}\right)$ was only about 0.8 bits in the small-range condition and 1.4 bits in the large-range condition. In many absolute identification experiments, $\mathrm{U}\left(\mathrm{R}_{n}: \mathrm{S}_{n}\right)$ is 2.0 bits or more (Garner, 1962; Miller, 1956). Since sequential dependencies are generally larger at lower levels of information transmission, it is possible

Table 3

Average Regression Coefficients and $R^{2}$ of Equations 3 and 4: Experiment 1

\begin{tabular}{|c|c|c|c|c|c|c|c|c|}
\hline \multirow[b]{3}{*}{ Sequence } & \multicolumn{8}{|c|}{ Equation } \\
\hline & \multicolumn{4}{|c|}{3} & \multicolumn{4}{|c|}{4} \\
\hline & $\alpha_{1}$ & $\alpha_{2}$ & $\alpha_{3}$ & $\mathrm{R}^{2}$ & $\beta_{1}$ & $\beta_{2}$ & $\rho$ & $\mathrm{R}^{2}$ \\
\hline \multicolumn{9}{|c|}{ Small Range } \\
\hline Feedback & $.802^{\dagger}$ & .045 & .076 & $.739^{\dagger}$ & $.802^{+}$ & $.110^{\dagger}$ & .083 & $.739^{4}$ \\
\hline No-feedback & $.807^{\star}$ & $-.199^{+}$ & .304 & $.747^{\dagger}$ & $.809^{+}$ & $.055^{\dagger}$ & $.312^{\dagger}$ & $.749^{\dagger}$ \\
\hline \multicolumn{9}{|c|}{ Large Range } \\
\hline Feedback & $.914^{+}$ & .049 & .004 & $.866^{\dagger}$ & $.914^{\dagger}$ & $.055^{\dagger}$ & .018 & $.866^{\dagger}$ \\
\hline No-feedback & $.931^{\dagger}$ & $-.183^{\dagger}$ & $.241^{\dagger}$ & $.881^{\dagger}$ & $.932^{+}$ & $.047^{*}$ & $.250^{\dagger}$ & $.882^{\dagger}$ \\
\hline
\end{tabular}


that the effects we observed in Experiment 1 would disappear at higher levels of performance. Because in previous studies (Mori, 1991; Mori \& Ward, 1992) we obtained more than 2.0 bits of information transmission for absolute identification of sound frequency, we used that as a stimulus modality. Although there are differences between intensity and frequency resolution (Mori \& Ward, 1992), sequential dependencies are generally similar for the two modalities (Mori, 1991).

Another purpose of this experiment was to examine how the effects of feedback depend on the number of stimuli used in the experiment in a way that might resolve an apparent discrepancy in the literature. For soundintensity resolution, Garner (1953) found that information transmission $\left[\mathrm{U}\left(\mathrm{R}_{n}: \mathrm{S}_{n}\right)\right]$ decreased but sequential effects $\left[\mathrm{U}\left(\mathrm{R}_{n}: \mathrm{S}_{n-1} \mid \mathrm{S}_{n}\right)\right]$ increased as the number of stimuli was increased. In contrast, Braida and Durlach (1972) argued that total sensitivity (summed $d^{\prime}$ ) for soundintensity resolution was a function of stimulus range and was unaffected by the number of stimuli when stimulus range was fixed. Also, for sound frequency, Mori (1991) found that as the number of stimuli was increased, information transmission was relatively constant but sequential dependencies increased in magnitude. Since total sensitivity and information transmission are supposed to be measuring the same thing, albeit with different assumptions, this apparent conflict is worth investigating further. Since feedback also affects both sensitivity and information transmission measures, the effects of feedback could also vary with the number of stimuli. However, we know of no previous experiment that has been done to examine the effects of feedback as a function of number of stimuli used. In this experiment, we used the sequence-alternating method with sets of 6,11 , and 16 tones.

\section{Method}

\section{Subjects}

Five students of the University of British Columbia, one of them the first author, participated in this experiment. Four were male and one was female, and none had any difficulty hearing the tones used as stimuli.

\section{Apparatus and Stimuli}

The apparatus was identical to that used in Experiment 1. Table 4 shows the frequencies of tones used as stimuli. Those stimuli were selected from a set of 16 frequencies that ranged from 100 to $8000 \mathrm{~Hz}$ in equidistant logarithmic steps. In order to prevent a loudness cue from aiding identification, each subject was asked to match the loudness of the tone of each frequency with the loudness of a $1000-\mathrm{Hz}, 60-\mathrm{dB}$ tone before the absolute identification experiment. The resulting matches determined tone intensities for that subject. The tone duration was $500 \mathrm{msec}$.

\section{Procedure}

The procedure was identical to that of Experiment 1, except for the number of tones to be judged and the number of trials in each feedback/no-feedback sequence. In each sequence, there were 36 trials for the 6-tone condition, 33 trials for the 11 -tone condition, and 32 trials for the 16-tone condition. Every subject participated in all conditions. The subjects had a 100-300-trial practice session before they started in each condition. Each subject made 60 judgments per tone for each condition, and there were 2 sessions for the 6-tone condition, 4 sessions for the 11 -tone condition, and 6 sessions for the 16-tone condition. The order of the conditions was randomized among the subjects.

\section{Results}

\section{Sensitivity Measures}

Figure 2 presents $d_{i, i+1}$ values in the feedback and nofeedback sequences for the three sets of stimuli used. The total sensitivities were 15.8 (feedback) and 14.9 (nofeedback) for the set of 6 stimuli, 14.4 (feedback) and 12.8 (no-feedback) for the set of 11 stimuli, and 15.6 (feedback) and 15.3 (no-feedback) for the set of 16 stimuli. The results are consistent with those of Experiment 1 , in that (1) there was no marked difference between $d^{\prime}$ values for the feedback sequences and those for the no-feedback sequences (feedback increased summed $d^{\prime}$ in the three conditions by $6 \%, 13 \%$, and $2 \%$, respectively), and (2) edge effects were observed for the three sets of stimuli. Although the values of $d^{\prime}$ for adjacent stimulus pairs were generally higher for the smaller number of stimuli, the total sensitivities showed no consistent change with the number of stimuli used. Since the frequency range of the stimuli did not vary in the present experiment, this result is in general agreement with the argument of Durlach and Braida (1972) that the number of stimuli does not affect total sensitivity.

\section{Multivariate Information Analysis}

Table 5 presents the results of the multivariate information analyses. The obtained amounts of $\mathrm{U}\left(\mathrm{R}_{n}: \mathrm{S}_{n}\right)$ (information transmission) were $1.8-2.2$ bits, and were comparable to those obtained in our previous studies (Mori, 1991; Mori \& Ward, 1992). For all stimulus sets, $\mathrm{U}\left(\mathrm{R}_{n}: \mathrm{S}_{n}\right)$ was slightly larger $(0.05-0.15$ bit, about $5 \%)$ in the feedback sequences than in the no-feedback sequences. Consistent with previous studies (Garner, 1953; Mori, 1991), the measures of sequential dependencies $\left[\mathrm{U}\left(\mathrm{R}_{n}: \mathrm{S}_{n-1} \mid \mathrm{S}_{n}\right)\right.$ and $\left.\mathrm{U}\left(\mathrm{R}_{n}: \mathrm{R}_{n-1} \mid \mathrm{S}_{n}, \mathrm{~S}_{n-1}\right)\right]$ increased with increasing number of stimuli, in both the feedback and the no-feedback sequences.

For all stimulus sets, the pattern of sequential dependencies in the feedback and no-feedback sequences was

Table 4

Frequencies of Tones Used as Stimuli in Experiment 2

\begin{tabular}{|c|c|c|c|c|c|c|c|c|c|c|c|c|c|c|c|c|}
\hline \multirow{2}{*}{$\begin{array}{c}\text { Number of } \\
\text { Stimuli }\end{array}$} & \multicolumn{16}{|c|}{ Frequency $(\mathrm{Hz})$} \\
\hline & 100 & 134 & 179 & 240 & 322 & 431 & 577 & 773 & 1035 & 1386 & 1857 & 2487 & 3330 & 4460 & 5973 & 8000 \\
\hline 6 & $\mathrm{x}$ & & & $x$ & & & $x$ & & & $\mathrm{x}$ & & & $\mathrm{x}$ & & & $\mathbf{x}$ \\
\hline 11 & $\mathrm{x}$ & $x$ & & $x$ & $\mathrm{x}$ & & $\mathrm{x}$ & $\mathrm{x}$ & & $\mathrm{x}$ & $x$ & & $x$ & $\mathrm{x}$ & & $\mathrm{x}$ \\
\hline 16 & $\mathrm{x}$ & $\mathrm{x}$ & $x$ & $x$ & $x$ & $x$ & $\mathrm{x}$ & $\mathrm{x}$ & $\mathrm{x}$ & $\mathrm{x}$ & $x$ & $x$ & $x$ & $x$ & $\mathrm{x}$ & $x$ \\
\hline
\end{tabular}

Note $-\mathrm{x}$ indicates the frequency of a tone used as a stimulus. 

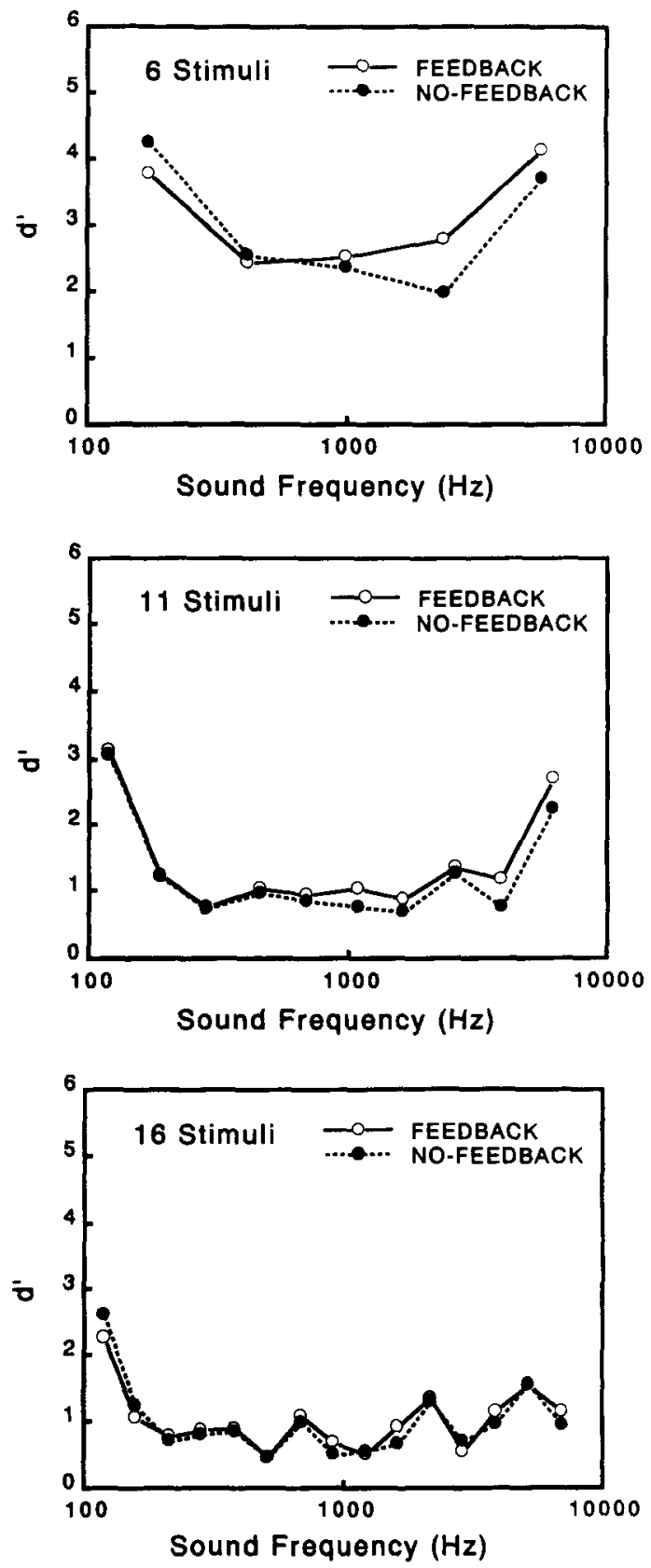

Figure 2. Sensitivity measures ( $d^{\prime}$ values) to adjacent pairs of stimuli in Experiment 2. similar to that of Experiment 1 , in that $\mathrm{U}\left(\mathrm{R}_{n}: \mathrm{S}_{n-1} \mid \mathrm{S}_{n}\right)$ was larger in the feedback sequences than in the no-feedback sequences, and in that $\mathrm{U}\left(\mathrm{R}_{n}: \mathrm{R}_{n-1} \mid \mathrm{S}_{n}, \mathrm{~S}_{n-1}\right)$ was smaller in the feedback sequences than in the no-feedback sequences. In addition, in the feedback sequences, $\mathrm{U}\left(\mathrm{R}_{n}: \mathrm{S}_{n-1} \mid \mathrm{S}_{n}\right)$ was always larger than $\mathrm{U}\left(\mathrm{R}_{n}: \mathrm{R}_{n-1} \mid \mathrm{S}_{n}, \mathrm{~S}_{n-1}\right)$, and the pattern was the opposite in the no-feedback sequences.

\section{Multiple Regression Analysis}

Tables 2 and 6 present the results of multiple regression analysis, which are also in general agreement with the results of Experiment 1 . The $\mathrm{R}^{2}$ increases showed the same pattern for all stimulus sets (Table 2). $\mathrm{R}^{2}$ increases by $\mathrm{S}_{n}$ were much larger than those by $\mathrm{S}_{n-1}$ and $\mathrm{R}_{n-1}$, and were slightly larger in the feedback sequences than in the no-feedback sequences. In the feedback sequences, $\mathrm{S}_{n-1}$ increased $\mathrm{R}^{2}$ more than $\mathrm{R}_{n-1}$ did, whereas the pattern was the opposite in the no-feedback sequences.

The regression coefficients and autoregressive parameters for Equations 3 and 4 (Table 6) showed a pattern similar to that of the $\mathrm{R}^{2}$ increases. The coefficients of $S_{n}\left(\alpha_{1}\right.$ and $\left.\beta_{1}\right)$ were slightly larger in the feedback sequences than in the no-feedback sequences. The measures of dependency on $\mathrm{R}_{n-1}\left(\alpha_{3}\right.$ and $\rho$ ) were much larger in the no-feedback sequences than in the feedback sequences. However, the coefficients of $S_{n-1}$ obtained for Equation 3 were all negative in the no-feedback sequences, and those obtained for Equation 4 showed no consistent difference between the feedback and the nofeedback sequences.

\section{Discussion}

There were two purposes of this experiment. One was to examine the generality of the results of Experiment 1 in an experiment in which the judgmental accuracy was high. In this experiment, the obtained amounts of $\mathrm{U}\left(\mathrm{R}_{n}: \mathrm{S}_{n}\right)$ were reasonably high and comparable to previous studies (e.g., Mori, 1991), and all the analyses yielded results quite similar to those of Experiment 1. The very slight improvement in performance (about $7 \%$ ) in the feedback sequences was much smaller than that observed in similar previous studies of frequency resolution (e.g., Siegel [1972] found 35\% improvement). The second purpose was to examine how the effects of feedback on absolute identification performance would

Table 5

Results of Multivariate Information Analysis: Experiment 2

\begin{tabular}{|c|c|c|c|c|}
\hline Sequence & $\mathrm{U}\left(\mathrm{R}_{n}\right)$ & $\mathrm{U}\left(\mathrm{R}_{n}: \mathrm{S}_{n}\right)$ & $\mathrm{U}\left(\mathrm{R}_{n}: \mathrm{S}_{n-1} \mid \mathrm{S}_{n}\right)$ & $\mathrm{U}\left(\mathbf{R}_{n}: \mathbf{R}_{n-1} \mid \mathbf{S}_{n}, \mathbf{S}_{n-1}\right)$ \\
\hline \multicolumn{5}{|c|}{6 Stimuli } \\
\hline Feedback & 2.584 & 2.051 & .049 & .013 \\
\hline No-feedback & 2.582 & 1.995 & .031 & .069 \\
\hline \multicolumn{5}{|c|}{11 Stimuli } \\
\hline Feedback & 3.458 & 2.001 & .168 & .153 \\
\hline No-feedback & 3.456 & 1.818 & .116 & .358 \\
\hline \multicolumn{5}{|c|}{16 Stimuli } \\
\hline Feedback & 3.992 & 2.204 & .239 & .199 \\
\hline No-feedback & 3.994 & 2.040 & .157 & .430 \\
\hline
\end{tabular}


Table 6

Average Regression Coefficients and $R^{2}$ of Equations 3 and 4: Experiment 2

\begin{tabular}{|c|c|c|c|c|c|c|c|c|}
\hline \multirow[b]{3}{*}{ Sequence } & \multicolumn{8}{|c|}{ Equation } \\
\hline & \multicolumn{4}{|c|}{3} & \multicolumn{4}{|c|}{4} \\
\hline & $\alpha_{1}$ & $\alpha_{2}$ & $\alpha_{3}$ & $\mathrm{R}^{2}$ & $\beta_{1}$ & $\beta_{2}$ & $\rho$ & $\mathrm{R}^{2}$ \\
\hline \multicolumn{9}{|c|}{6 Stimuli } \\
\hline Feedback & $.979^{\dagger}$ & -.020 & .029 & $.962^{\dagger}$ & $.979^{\prime}$ & .010 & .025 & $.962^{\dagger}$ \\
\hline No-feedback & $.965^{t}$ & $-.172^{+}$ & $.191^{\dagger}$ & $.956^{*}$ & $.965^{t}$ & $.013^{\circ}$ & $.198^{\dagger}$ & $.957^{+}$ \\
\hline \multicolumn{9}{|c|}{11 Stimuli } \\
\hline Feedback & $.959^{+}$ & .027 & .002 & $.936^{+}$ & $.959^{+}$ & .029 & .018 & $.936^{\dagger}$ \\
\hline No-feedback & $.940^{\dagger}$ & $-.182^{+}$ & $.212^{\ddagger}$ & $.912^{4}$ & $.941^{*}$ & $.019^{7}$ & $.219^{\dagger}$ & $.912^{+}$ \\
\hline \multicolumn{9}{|c|}{16 Stimuli } \\
\hline Feedback & $.974^{\dagger}$ & .003 & .010 & $.949^{\circ}$ & $.974^{*}$ & .013 & .016 & $.949^{\ddagger}$ \\
\hline No-feedback & $.956^{\dagger}$ & $-.175^{+}$ & $.208^{\dagger}$ & $.943^{\dagger}$ & $.955^{t}$ & $.024^{*}$ & $.209^{\dagger}$ & $.943^{+}$ \\
\hline
\end{tabular}

depend on the number of stimuli used. The results of this experiment clearly showed that the effects of feedback on performance, such as judgmental accuracy and sequential dependency on $\mathrm{S}_{n-1}$ and $\mathrm{R}_{n-1}$, remained the same for all the numbers of stimuli used in this experiment, although the overall size of sequential dependencies increased with increasing number of stimuli for both feedback and no-feedback sequences. Thus, neither accuracy nor the number of stimuli interact with feedback in its effects on sequential dependencies.

\section{EXPERIMENT 3}

The purpose of this experiment was to further investigate the effects of number of stimulus/response categories on sequential dependencies in the absolute-identification task. In Experiment 1, increasing the stimulus range (and coincidently the spacing between adjacent intensities) decreased the size of the sequential dependencies (see also Braida \& Durlach, 1972; Luce et al., 1976). In Experiment 2 , different numbers of tones were spaced over a fixed frequency range so that the frequency spacing between the tones decreased with increasing number of tones in the stimulus set (see Table 4). Thus, the finding of Experiment 2 that the size of sequential dependencies increased with increasing number of stimulus/response categories could be attributed to the stimulus spacing, the number of stimulus/response categories, or both.

An early study by Eriksen and Hake (1955) provided the basis for our investigation of this question. They found that the number of response categories affected identification of square size more than the number of stimuli did. In their experiment, the subjects first participated in an ordinary absolute identification task with feedback, in which the number of stimuli was equal to the number of response categories. In a subsequent session, the subjects were told that the task was identical, except that no feedback would be given. However, although the number of response categories remained the same, a different number of stimuli was used. The stim- ulus spacing was also manipulated. There were three important results in the study. First, doubling the stimulus spacing had only a small effect on $\mathrm{U}\left(\mathrm{R}_{n}: \mathrm{S}_{n}\right)$. Second, the amount of response information $\left[\mathrm{U}\left(\mathrm{R}_{n}\right)\right]$ was close to its maximum value (logarithm [base 2] of the number of response categories), irrespective of the number of stimuli used. This meant that the subjects had a strong tendency to use all responses permitted, even if the number of stimuli used was fewer than that of responses. Third, when the number of response categories was equal to or greater than the number of stimuli, $\mathrm{U}\left(\mathrm{R}_{n}: \mathrm{S}_{n}\right)$ was the same irrespective of the number of stimuli. These results imply that the subject's performance was more dependent on the number of response categories than on the number of stimuli. Although Eriksen and Hake (1955) did not analyze sequential dependencies in their experiment, it is possible that the number of response categories also has a dominant effect on the sequential dependencies.

Following Eriksen and Hake (1955), in Experiment 3 we used the sequence-alternating method, with stimuli and procedure identical to those of Experiment 2, except that the number of stimuli used was fewer than that of response categories in the no-feedback sequences. If the number of response categories used has a dominant effect on the subject's performance, this experiment should replicate Eriksen and Hake's (1955) results, and the sequential dependencies should be more dependent on the number of response categories than on the number of stimuli used.

\section{Method}

\section{Subjects}

Six students of the University of British Columbia participated in this experiment. Five were male and one was female, and none had any difficulty hearing the tones used as stimuli.

\section{Apparatus and Stimuli}

The apparatus and stimuli were identical to those used in Experiment 2. As in Experiment 2, each subject matched the loudness of each of 16 tones with that of a $1000-\mathrm{Hz}, 60-\mathrm{dB}$ tone to produce 
60-phon intensities for all stimulus frequencies, and the resulting matches were used as stimuli for that subject.

\section{Procedure}

The procedure was identical to that of Experiment 2, except that in the no-feedback sequences, the number of tones used was fewer than the number of response categories. There were three conditions with respect to the combination of the number of tones and response categories in the no-feedback sequences: Condition 1 6 tones and 11 response categories; Condition $2-11$ tones and 16 response categories; and Condition 3--6 tones and 16 response categories. As mentioned before, the same number of tones and response categories was used in the feedback sequence in each condition; 11 tones were used in the feedback sequence in Condition 1 , and 16 tones were used in Conditions 2 and 3. The sets of tones used in the three conditions were identical to those of Experiment 2 (Table 4). The number of trials in the feedback and nofeedback sequences was made roughly the same- 33 trials in the feedback sequence and 36 trials in the no-feedback sequence in Condition 1; 32 trials and 33 trials in Condition 2; and 32 trials and 36 trials in Condition 3 . In all conditions, the feedback sequence was given first, and was followed by the no-feedback sequence. The two sequences were repeated five times in one session, so that one session consisted of 325-345 trials.

The subjects were instructed that the same number of tones was used in both the feedback and no-feedback sequences, and that they should keep their performance consistent in both sequences. During the experimental sessions, none of them noticed that the actual number of stimuli presented was smaller in the no-feedback sequences than in the feedback sequences.

Every subject performed in all three conditions. Each subject made 60 judgments per tone in the no-feedback sequences in each condition. The order of the conditions was randomized across the subjects.

\section{Results}

\section{Sensitivity Measures}

Figure 3 presents $d_{i, i+1}$ values in the feedback and nofeedback sequences for the three conditions. [In the nofeedback sequence of Condition $3, d_{1,2}^{\prime}$ was not calculated because all $\operatorname{Pr}(\mathrm{R} \geq j \mid \mathrm{S}=1)$ values were outside .01 and .99. Probably because of this missing value of $d_{1,2}^{\prime}$, the Newton-Raphson method did not yield reasonable maximum-likelihood estimates for these data. Therefore, the initial estimates calculated from the confusion matrix are reported for this sequence of Condition 3; see the Method section of Experiment 1]. In Conditions 1 and 3 , the $d^{\prime}$ values were higher in the no-feedback sequence than in the feedback sequence, and this is probably due to the much larger stimulus spacing in the nofeedback sequence. In Condition 2, the $d^{\prime}$ values in the no-feedback sequence were not much different from those in the feedback sequence. Resolution edge effects were observed in all three conditions, especially at the lower frequencies, and were more apparent in the feedback sequences than in the no-feedback sequences.

The total sensitivities were 13.6 (feedback) and 13.0 (no-feedback) in Condition 1, 15.1 (feedback) and 14.9 (no-feedback) in Condition 2, and 10.9 (feedback) and 10.6 (no-feedback) in Condition 3 (initial estimates and omitting $d_{1.2}^{\prime}$ for both sequences in this condition, since it could not be calculated for the no-feedback sequence). As observed in Experiments 1 and 2, they did not show
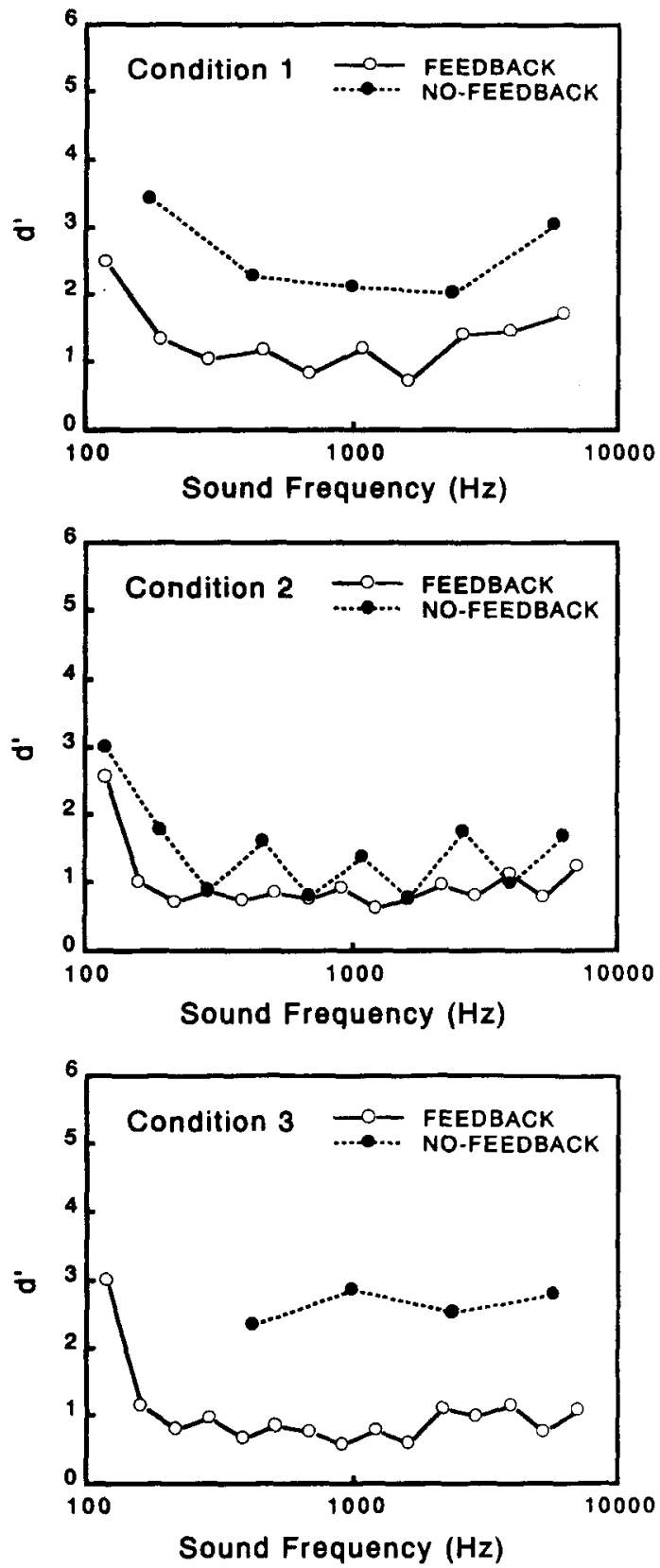

Figure 3. Sensitivity measures ( $d^{\prime}$ values) to adjacent pairs of stimuli in Experiment 3. Note that stimulus spacing differs for each pair of curves.

a marked difference between the feedback and the nofeedback sequences. Again, this result is consistent with those of Braida and Durlach (1972), in that total sensitivity was unaffected by the number of stimuli and/or response categories when the stimulus range was constant.

\section{Multivariate Information Analysis}

Multivariate information analysis (and the correction of overestimation method) ${ }^{4}$ was performed on data from the no-feedback sequences for each of the three conditions. Table 7 presents the results. We also used the re- 
Table 7

Results of Multivariate Information Analysis: No-Feedback Sequences of Experiment 3

\begin{tabular}{ccccc}
\hline Condition & $\mathrm{U}\left(\mathrm{R}_{n}\right)$ & $\mathrm{U}\left(\mathrm{R}_{n}: \mathrm{S}_{n}\right)$ & $\mathrm{U}\left(\mathrm{R}_{n}: \mathrm{S}_{n+1} \mid \mathrm{S}_{n}\right)$ & $\mathrm{U}\left(\mathrm{R}_{n}: \mathrm{R}_{n-1} \mid \mathrm{S}_{n}, \mathrm{~S}_{n-1}\right)$ \\
\hline $\mathrm{l}$ & 3.343 & 1.999 & .048 & .245 \\
2 & 3.973 & 2.089 & .117 & .413 \\
3 & 3.824 & 2.126 & .076 & .257 \\
\hline
\end{tabular}

sults of the no-feedback sequences in Experiment 2, since these were conditions in which the same numbers of tones and response categories were used. Figure 4 presents the information measures of this experiment, along with those of Experiment 2 for comparison.

The obtained amounts of $\mathrm{U}\left(\mathrm{R}_{n}\right)$ of this experiment were close to their maximum values ( 3.459 bits for 11 response categories and 4 bits for 16 response categories). Figure 4 shows that $\mathrm{U}\left(\mathrm{R}_{n}\right)$ depended more on the number of response categories than on the number of tones used. When $\mathrm{U}\left(\mathrm{R}_{n}\right)$ was plotted against the number of tones used (left panel), it is only when 6 tones were used that $\mathrm{U}\left(\mathrm{R}_{n}\right)$ was about 0.15 bits smaller than would have been the case if all 11 or 16 response categories had been used equally often.

The estimates of $\mathrm{U}\left(\mathrm{R}_{n}: \mathrm{S}_{n}\right)$ (information transmission) were about $1.8-2.1$ bits. $\mathrm{U}\left(\mathrm{R}_{n}: \mathrm{S}_{n}\right)$ slightly decreased as the number of tones used increased (left panel), whereas with a fixed number of tones (right panel), it was $0.2-0.3$ bits larger for 16 response categories than for 6 or 11 response categories. This was caused by relatively poor performance in the 11-tone-11-response condition (from Experiment 2).

Regardless of the number of tones or response categories, $\mathrm{U}\left(\mathrm{R}_{n}: \mathrm{S}_{n-1} \mid \mathrm{S}_{n}\right)$ was smaller than $\mathrm{U}\left(\mathrm{R}_{n}: \mathrm{R}_{n-1} \mid\right.$ $\mathrm{S}_{n}, \mathrm{~S}_{n-1}$ ) (Table 7). In Figure 4, with a fixed number of response categories (left panel), $\mathrm{U}\left(\mathrm{R}_{n}: \mathrm{S}_{n-1} \mid \mathrm{S}_{n}\right)$ steadily increased as the number of tones increased. When 6 tones were used (right panel), $\mathrm{U}\left(\mathrm{R}_{n}: \mathrm{S}_{n-1} \mid \mathrm{S}_{n}\right)$ increased with an increasing number of response categories. With 11 tones, there was no increase of $\mathrm{U}\left(\mathrm{R}_{n}: \mathrm{S}_{n-1} \mid \mathrm{S}_{n}\right)$ from 11 to 16 response categories. $\mathrm{U}\left(\mathrm{R}_{n}: \mathrm{R}_{n-1} \mid \mathrm{S}_{n}, \mathrm{~S}_{n-1}\right)$ increased as the number of tones and/or response categories increased (bottom panels of Figure 4).

We conducted multiple regression analysis in the same manner that we did in Experiments 1 and 2, and the $\mathrm{R}^{2}$ increase and the coefficients showed patterns similar to those of the previous experiments. However, we do not report those results in detail because when we plotted them in the same way as we plotted the information measures in Figure 4, they showed no consistent change with the number of tones or the number of response categories and did not aid our understanding of the results.

\section{Discussion}

This experiment, together with Experiment 2, replicated Eriksen and Hake's (1955) results, but with the effects of feedback on judgmental accuracy controlled and with the manipulation of feedback occurring within a single session. Whereas the $d^{\prime}$ values for adjacent pairs of stimuli were larger in the feedback sequences than in the no-feedback sequences, showing the effect of stimulus spacing, the total sensitivities (summed $d^{\prime}$ ) were not different between the feedback and no-feedback se-
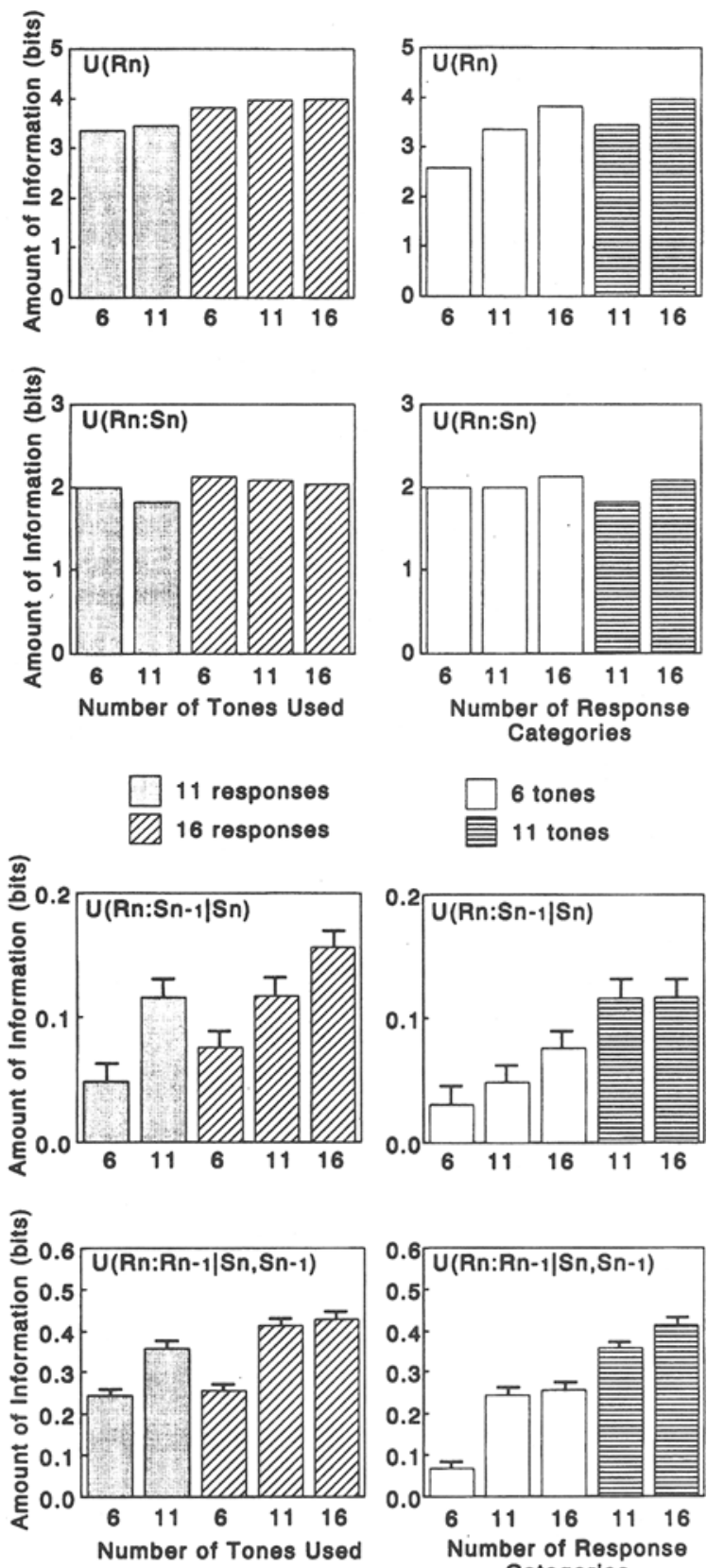

Figure 4. Estimates of information measures in Experiment 3, plotted both as a function of the number of tones used with a fixed number of response categories (left panel) and as a function of the number of response categories with a fixed number of tones used (right panel). Error bars of 0.015 bits are shown for $U\left(R_{n}: S_{n-1} \mid S_{n}\right)$ and $U\left(R_{n}: R_{n, 1} \mid S_{n}, S_{n-1}\right)$; they are too small to be visible for $U\left(R_{n}\right)$ and $\mathrm{U}\left(\mathrm{R}_{n}: \mathrm{S}_{n}\right)$. 
quences. $\mathrm{U}\left(\mathrm{R}_{n}\right)$ was close to its maximum value, and depended more on the number of response categories than on the number of stimuli, and $\mathrm{U}\left(\mathrm{R}_{n}: \mathrm{S}_{n}\right)$ was roughly constant for all the numbers of stimuli and response categories in the two experiments. These results suggest that the subjects of this experiment also had a tendency to use equally often all responses permitted even if the number of stimuli was fewer than the number of responses, at least as much as the subjects did in previous experiments in which the number of stimuli was equal to the number of response categories.

The results of the multivariate information analysis (Figure 3) showed that both the number of stimuli and the number of response categories had some effects on the sequential dependency on $\mathrm{S}_{n-1}$ and $\mathrm{R}_{n-1}$. Although these results may be of interest, it is hard to interpret them because the effects were subtle. In order to obtain sufficiently large effects to interpret unambiguously, it may be necessary to run an experiment in which the differences between the number of response categories and the number of stimuli used are larger than those used in Experiment 3 (e.g., 6 stimuli and 25 response categories).

\section{GENERAL DISCUSSION}

\section{Effects of Feedback on Judgmental Accuracy}

As predicted, the sequence-alternating method was very effective in keeping judgmental accuracy at roughly the same level in the feedback and no-feedback sequences. This suggests that in the sequence-alternating method, subjects re-established the memory of context of the stimulus set (Braida \& Durlach, 1972, 1988) in each feedback sequence and were able to maintain it throughout the subsequent no-feedback sequence.

The results of Experiment 1 are consistent with Braida and Durlach's (1972, 1988; Purks et al., 1980) model. We found the resolution edge effect in both the feedback and the no-feedback sequences of the large-range condition, and it could be argued that feedback strengthened the long-term memory for perceptual anchors in the feedback sequence and that the effect persisted in the nofeedback sequence. The model predicts no resolution edge effect in the small-range condition, and that prediction was confirmed in both the feedback and the no-feedback sequences.

Although Braida and Durlach's $(1972,1988)$ model was not intended to apply to frequency resolution, a straightforward extension of it does seem to be consistent with the results of Experiments 2 and 3. There were minimal effects of feedback on frequency resolution in these experiments, indicating that long-term memory for perceptual anchors was strengthened in the feedback sequences and maintained during the no-feedback sequences. Resolution edge effects were also observed in both experiments, and they too were unaffected by feedback (recall that the $d^{\prime}$ for the lowest pair in Condition 3 of Experiment 3 could not be calculated because of perfect performance). Further, the total sensitivity was unaffected by the number of stimuli and/or response cate- gories used in those experiments. In general, the simple resolution results of all three experiments can be accounted for by a modification of Braida and Durlach's model. However, the theory of the response process in that model does not explain sequential dependencies in the responses themselves, only the overall effects of variables such as stimulus range and experimental method on intensity (or frequency) resolution. An additional theory is needed to deal with the effects of feedback on sequential dependencies.

\section{Effects of Feedback on Sequential Dependencies}

In Experiments 1 and 2, the stimulus-response correlation, measured by $\mathrm{U}\left(\mathrm{R}_{n}: \mathrm{S}_{n}\right)$, remained roughly constant in the feedback and no-feedback sequences. Therefore, those two experiments should reveal pure effects of feedback on measures of sequential dependencies. The clear finding in both experiments was that in the feedback sequences, $S_{n-1}$ affected $R_{n}$ more than it did in the no-feedback sequences, while $\mathrm{R}_{n-1}$ affected $\mathrm{R}_{n}$ more in the no-feedback sequences than it did in the feedback sequences. Moreover, in Experiment 2, $\mathrm{S}_{n-1}$ affected $\mathrm{R}_{n}$ more than $R_{n-1}$ did in the feedback sequences, while $\mathrm{R}_{n-1}$ affected $\mathrm{R}_{n}$ more than $\mathrm{S}_{n-1}$ did in the no-feedback sequences.

It is important to note that both the amount of information transmission $\left[\mathrm{U}\left(\mathrm{R}_{n}: \mathrm{S}_{n}\right)\right.$; Experiment 1] and the number of stimulus/response categories (Experiment 2) consistently affect the overall size of sequential dependencies (this study; Garner, 1953; McGill, 1957; Mori, 1989; Ward \& Lockhead, 1971). Giving feedback seemed to affect both the form and the size of sequential dependencies in the previous studies (Mori, 1989; Ward \& Lockhead, 1970, 1971), because the stimulus/response correlation was different in the sessions with feedback from that in the sessions without feedback. However, once that correlation is carefully controlled, as in the present study, the presence or absence of feedback can be seen to affect only the form of sequential dependencies.

The present results have some implications for a recent model of the response process in absolute identification (Treisman, 1985; Treisman \& Williams, 1984). Treisman (1985) proposed a criterion-setting model that is based on a Thurstonian approach, like Braida and Durlach's $(1972,1988)$ model. In the criterion-setting model, sensations of presented stimuli are evaluated with respect to response criteria, as in Thurstone's (1927) law of categorical judgment and in signal detection theory. However, unlike those theories, there are assumed to be three mechanisms responsible for setting response criteria in the absolute identification task. One of them, the reference system, sets the initial positions of response criteria in the task. This mechanism resembles that of signal detection theory and could easily be modeled by Braida and Durlach's theory of intensity resolution. The other two mechanisms, stabilization and tracking, shift the response criteria on a trial-by-trial basis; stabilization shifts all criteria toward the sensation of the previous stimulus, while tracking shifts all criteria away from 
the previous response. These mechanisms are provided to allow the decision-maker to be sensitive to momentarily changing stimulus probabilities while still keeping the response criteria in a useful range. They resemble the mechanisms proposed less formally by others (e.g., Parducci, 1965; Ward \& Lockhead, 1971) for similar reasons. The final criteria used to identify a stimulus on a current trial are effectively obtained by shifting their locations on the previous trial by a linear combination of amounts indicated by stabilization and tracking. In this theory, the operation of stabilization and tracking causes the observed sequential dependencies of $\mathrm{R}_{n}$ on $\mathrm{S}_{n-1}$ and on $\mathrm{R}_{n-1}: \mathrm{R}_{n}$ (given to $\mathrm{S}_{n}$ ) to be biased away from $\mathrm{S}_{n-1}$ by the shift of criteria toward that stimulus (stabilization), and toward $R_{n-1}$ by the shift of criteria away from that response (tracking).

Treisman and Williams (1984) assumed that giving feedback affects only the tracking mechanism. In the presence of feedback, tracking is active only if a previous response is equal or close to the feedback value associated with it. In the absence of feedback, all responses are effective for tracking. This assumption leads directly to a prediction of a larger dependency of $\mathbf{R}_{n}$ on $\mathrm{R}_{n-1}$ in the absence of feedback than in the presence of feedback. Interestingly, it also implies a relatively larger dependency of $\mathrm{R}_{n}$ on $\mathrm{S}_{n-1}$ in the presence of feedback than in its absence. This is because as long as performance is less than perfect and equal in the two conditions, the effect of stabilization on response criteria will be relatively larger when feedback reduces the number of trials on which tracking is active. The effect of feedback on the form of sequential dependencies in Experiments 1 and 2 clearly confirms these predictions. Moreover, the theory predicts that the better a subject's performance in feedback conditions (i.e., the more closely responses correspond to feedbacks), the less often the tracking mechanism should be active and the larger should be the effects of $S_{n-1}$ relative to those of $R_{n-1}$. A comparison of the relative sizes of the effects of $S_{n-1}$ and $R_{n-1}$ across Experiments 1 and 2 also confirms this prediction. The ratio of $\mathrm{U}\left(\mathrm{R}_{n}: \mathrm{S}_{n-1} \mid \mathrm{S}_{n}\right)$ to $\mathrm{U}\left(\mathrm{R}_{n}: \mathrm{R}_{n-1} \mid \mathrm{S}_{n}, \mathrm{~S}_{n-1}\right)$ in the feedback conditions varies directly with information transmission: in Experiment 2, when $\mathrm{U}\left(\mathrm{R}_{n}: \mathrm{S}_{n}\right)$ was about 2.0 bits, the ratio ranges from 3.77 to 1.1 , while in $\mathrm{Ex}$ periment 1 , when $\mathrm{U}\left(\mathrm{R}_{n}: \mathrm{S}_{n}\right)$ was about 1.4 bits, the ratio was 0.64 , and when it was about 0.87 bits, the ratio was 0.42. In Experiment 2, where the tracking mechanism should be least active, the greater-than- 1 ratio indicates that the effects of $\mathrm{S}_{n-1}$ dominated those of $\mathrm{R}_{n-1}$. Interestingly, this ratio also varies with information transmission in the no-feedback conditions (from 0.23 in the small-range condition of Experiment 1 to 0.32 in the large-range condition, to an average of 0.37 in Experiment 2), indicating that there may be some variation in the weighting of tracking with performance happening there as well.

However, it is important to note that the function of feedback assumed in the criterion-setting model is on a trial-by-trial basis. The present study has shown that giv- ing feedback also affects judgmental accuracy, and that the effect lasts at least as long as about 30 trials in which feedback is no longer present (no-feedback sequences). The criterion-setting model does incorporate a mechanism by which feedback can have such a long-lasting effect on accuracy. In that model, the reference system specifies optimum locations for the criteria relative to the distributions of sensations given rise to by the repeated presentations of the different stimulus intensities (or frequencies) using expectations formed about the present judgment situation and long-term memories of similar stimuli. Trial-by-trial feedback could affect the reference system by decreasing the variability of these sensation distributions, and this effect could persist for some number of subsequent trials without feedback. This is equivalent to asserting that feedback strengthens the long-term memory of context of a stimulus set within Braida and Durlach's $(1972,1988)$ model. Since both Braida and Durlach's and Treisman's (1985) models are described within a Thurstonian framework, it may be possible that the former model can be completely incorporated into the latter, possibly in place of the reference system. Such a unified model should be able to explain most findings in absolute identification, including the finding of effects of feedback on the form of sequential dependencies.

\section{REFERENCES}

Braida, L. D., \& Durlach, N. I. (1972). Intensity perception: II. Resolution in one-interval paradigms. Journal of the Acoustical Society of America, 51, 483-502.

Braida, L. D., \& Durlach, N. I. (1988). Peripheral and central factors in intensity perception. In G. M. Edelman, W. E. Gall, \& W. M. Cowan (Eds.), Auditory function (pp. 559-583). New York: Wiley.

Chase, S., Bugnacki, P., Braida, L. D., \& Durlach, N. I. (1983). Intensity perception: XII. Effect of presentation probability on absolute identification. Journal of the Acoustical Society of America, 73, 279-284.

DECARLo, L. T. (1992). Intertrial interval and sequential dependencies in magnitude scaling. Journal of Experimental Psychology: Human Perception \& Performance, 18, 1080-1088.

DeCarlo, L. T., \& Cross, D. V. (1990). Sequential effects in magnitude scaling: Models and theory. Journal of Experimental Psychology: General, 119, 375-396.

ERIKSEN, C. W. (1958). Effects of practice with or without correction on discriminative learning. American Journal of Psychology, 69, 350-358.

Eriksen, C. W., \& HaKe, H. W. (1955). Absolute judgments as a function of stimulus range and number of stimulus and response categories. Journal of Experimental Psychology, 49, 323-332.

GARNER, W. R. (1953). An informational analysis of absolute judgments of loudness. Journal of Experimental Psychology, 46, 373380 .

GARNER, W. R. (1962). Uncertainty and structure as psychological concepts. New York: Wiley.

Green, D. M., LuCE, R. D., \& DunCan, J. E. (1977). Variability and sequential effects in magnitude production and estimation of auditory intensity. Perception \& Psychophysics, 22, 450-456.

Holland, M. K., \& Lockhead, G. R. (1968). Sequential effects in absolute judgments of loudness. Perception \& Psychophysics, 3, 409414

Houtsma, A. J. M. (1983). Estimation of mutual information from limited experimental data. Journal of the Acoustical Society of America, 74, 1626-1629.

JeSTEADT, W., LuCE, R. D., \& GREEN, D. M. (1977). Sequential effects 
in judgments of loudness. Journal of Experimental Psychology: Human Perception \& Performance, 3, 92-104.

KRIPPENDORFF, K. (1986). Information theory: Structural model for qualitative data. Beverly Hills, CA: Sage.

LippmanN, R. P., Braida, L. D., \& Durlach, N. I. (1976). Intensity perception: V. Effect of payoff matrix on absolute identification. Journal of the Acoustical Society of America, 59, 129-134.

LuCE, R. D., GREEN, D. M., \& Weber, D. L. (1976). Attention bands in absolute identification. Perception \& Psychophysics, 20, 49-54.

MCGILL, W. J. (1954). Multivariate information transmission. Psychometrika, 19,97-116.

MCGILL, W. J. (1957). Serial effects in auditory threshold judgments. Journal of Experimental Psychology, 53, 297-303.

MCNicol, D. (1980). Multiple regression analysis of sequential effects in loudness judgments. In R. S. Nickerson (Ed.), Attention and performance VIII (pp.179-196). Hillsdale, NJ: Erlbaum.

MiLleR, G. A. (1956). The magical number seven plus or minus two: Some limits on our capacity for processing information. Psychological Review, 65, 81-97.

MoRI, S. (1989). A limited-capacity response process in absolute identification. Perception \& Psychophysics, 46, 167-173.

MORI, S. (1991). Dynamic information model of identification performance. Unpublished doctoral dissertation, University of British Coiumbia, Vancouver.

MoRI, S. (1994). Response process model of sequential dependencies in an absolute identification task. Manuscript submitted for publication.

MORI, S., \& WARD, L. M. (1990). Unmasking the magnitude estimation response. Canadian Journal of Psychology, 44, 58-68.

MORI, S., \& WARD, L. M. (1992). Intensity and frequency resolution: Masking of absolute identification, and fixed and roving discrimination. Journal of the Acoustical Society of America, 91, 246-255.

PARDucci, A. (1965). Category judgment: A range-frequency model. Psychological Review, 72, 407-418.

Purks, S. R., Callahan, D. J., Braida, L. D., \& Durlach, N. I. (1980). Intensity perception: X. Effect of preceding stimulus on identification performance. Journal of the Acoustical Society of America, 67, 634-637.

Schifferstein, H. N. J., \& Frujters, J. E. R. (1992). Contextual and sequential effects on judgments of sweetness intensity. Perception \& Psychophysics, 52, 243-255.

SIEGEL, W. (1972). Memory effects in the method of absolute judgment. Journal of Experimental Psychology, 94, 121-131.

Thurstone, L. L. (1927). A law of comparative judgment. Psychological Review, 34, 273-286.

TREISMAN, M. (1985). The magical number seven and some other features of category scaling: Properties of a model for absolute judgment. Journal of Mathematical Psychology, 29, 175-230.

Treisman, M., \& Williams, T. C. (1984). A theory of criterion setting with an application to sequential dependencies. Psychological Review, 91, 68-111.

WARD, L. M. (1979). Stimulus information and sequential dependencies in magnitude estimation and cross-modality matching. Journal of Experimental Psychology: Human Perception \& Performance, $\mathbf{5}$ 444-459.

WARD, L. M. (1982). Mixed-modality psychophysical scaling: Sequential dependencies and other properties. Perception \& Psy. chophysics, 31, 53-62.

WARD, L. M. (1985). Mixed-modality psychophysical scaling: Interand intramodality sequential dependencies as a function of lag. Perception \& Psychophysics, 38, 512-522.

WARD, L. M. (1986). Mixed-modality psychophysical scaling: Double cross-modality matching for "difficult" continua. Perception \& Psychophysics, 39, 407-417.

WARD, L. M. (1987). Remembrance of sounds past: Memory and psychophysical scaling. Journal of Experimental Psychology: Human Perception \& Performance, 13, 216-227.

WARD, L. M., \& LOCKHEAD, G. R. (1970). Sequential effects and memory in category judgments. Joumal of Experimental Psychology, 84, 27-34

WARd, L. M., \& Lockhead, G. R. (1971). Response system processes in absolute judgment. Perception \& Psychophysics, 9, 73-78.
WeSt, R., \& WARD, L. M. (1994). Constrained scaling. In L. M. Ward (Ed.), Fechner day '94 (pp. 225-230). Vancouver, Canada: International Society for Psychophysics.

\section{NOTES}

1. After 30 trials, accuracy would be expected to decrease gradually to an asymptotic no-feedback level as the subject's memory of the context deteriorates. We did not explore this method further in these experiments, since the procedure described was sufficient to fix performance, but other variants would be expected to produce interesting and useful results. In particular, it would be interesting to know just how long the effects of a sequence of feedback trials on memory for context can last. A variation of this procedure was used by West and Ward (1994) to train people in the use of a particular scale for loudness.

2. We thank Neil A. Macmillan for generously calculating the maximum-likelihood estimates of the $d_{i, i+1}^{\prime}$ values, using the method of Lippmann et al. (1976), based on our confusion matrices and our initial estimates.

3 . In the simulations, response sequences were generated from a combination of three 2-dimensional matrices that represented a joint probability distribution of current stimulus and response category $\left(\mathrm{S}_{n}\right.$ and $\mathrm{R}_{n}$ ), previous stimulus and current response category $\left(\mathrm{S}_{n-1}\right.$ and $\mathrm{R}_{n}$ ), and previous response and current response category $\left(\mathrm{R}_{n-1}\right.$ and $\mathrm{R}_{n}$ ). The standard deviations of these distributions were manipulated as parameters to change the size of the effects on $R_{n}$ of $S_{n}, S_{n-1}$, and $\mathrm{R}_{n-1}$, respectively. The stimulus sequence was randomized and each stimulus category was used equally often in the same way as was done in the present experiments. Simulations were repeated 10 times for each combination of parameters and averaged. This simulation method has an advantage over other methods in that information measures $\left[\mathrm{U}\left(\mathrm{R}_{n}: \mathrm{S}_{n}\right), \mathrm{U}\left(\mathrm{R}_{n}: \mathrm{S}_{n-1} \mid \mathrm{S}_{n}\right), \mathrm{U}\left(\mathrm{R}_{n}: \mathrm{R}_{n-1} \mid \mathrm{S}_{n}, \mathrm{~S}_{n-1}\right)\right]$ of the simulation data can be compared with their theoretical values that are calculated from the probability dist ributions of the matrices used in the simulation. The results showed that the asymptotic values of the simulated information measures generally approached their theoretical values (for more details, see Mori, 1991).

4. The simulations used to correct the information measures of this experiment were done with the same combinations of the number of stimuli and response categories as the combinations used in the experiment (e.g., 6 stimuli and 11 response categories in Condition 1).

\section{APPENDIX}

The amount of information transmission from $\mathrm{S}_{n}$ to $\mathrm{R}_{n}$ is defined by

$$
\mathrm{U}\left(\mathrm{R}_{n}: \mathrm{S}_{n}\right)=\mathrm{U}\left(\mathrm{S}_{n}\right)+\mathrm{U}\left(\mathrm{R}_{n}\right)-\mathrm{U}\left(\mathrm{S}_{n}, \mathrm{R}_{n}\right),
$$

where $\mathrm{U}\left(\mathrm{S}_{n}\right)=-\sum p_{i} \log _{2} p_{r} . \mathrm{U}\left(\mathrm{R}_{n}\right)=-\sum p_{. j} \log _{2} p_{j}$, and $\mathrm{U}\left(\mathrm{S}_{n}, \mathrm{R}_{n}\right)=-\sum p_{i j} \log _{2} p_{i j}$, and where $p_{i}$ and $p_{. j}$ are the probability of Stimulus Category $i$ and of Response Category $j$, respectively, and $p_{i j}$ is the joint probability of Stimulus Category $i$ and Response Category $j . \mathrm{U}\left(\mathrm{S}_{n}\right)$ and $\mathrm{U}\left(\mathrm{R}_{n}\right)$ measure the amount of information of $\mathrm{S}_{n}$ and of $\mathrm{R}_{n}$, respectively, and $\mathrm{U}\left(\mathrm{R}_{n}, \mathrm{~S}_{n}\right)$ measures the amount of joint information of $S_{n}$ and $R_{n}$.

Let us denote $M$ as the number of total observations in a data set, $m_{i}$ as the number of presentations of Stimulus Category $i$, $m_{j}$ as the number of occurrences of Response Category $j$, and $m_{i j}$ as the number of joint occurrences of Stimulus Category $i$ and Response Category $j$; and let us define $T$ as $\log _{2} M, T_{i}$ as $(1 / M) \sum m_{i} \log _{2} m_{i}, T_{j}$ as $(1 / M) \sum m_{j} \log _{2} m_{j}$, and $T_{i j}$ as $(1 / M)$ $\sum m_{i j} \log _{2} m_{i}$, so we can rewrite Equation $\mathrm{A} 1$ as

$$
\mathrm{U}\left(\mathrm{R}_{n}: \mathrm{S}_{n}\right)=T-T_{i}-T_{j}+T_{i j}
$$

(McGill, 1954 ).

Next, we extend this equation to include three inputs, $\mathrm{S}_{n}$, $\mathrm{S}_{n-1}, \mathrm{R}_{n-1}$. We denote $m_{k}$ and $m_{l}$ as the number of occurrences 
of Category $k$ of the previous stimulus $\left(\mathrm{S}_{n-1}\right)$ and the number of occurrences of Category $l$ of the previous response $\left(\mathrm{R}_{n-1}\right)$, respectively. We have the amount of information transmission from $\mathrm{S}_{n-1}$ to $\mathrm{R}_{n}$ as

$$
\mathrm{U}\left(\mathrm{R}_{n}: \mathrm{S}_{n-1}\right)=T-T_{j}-T_{k}+T_{j k} .
$$

But since this measure includes the effect of $\mathrm{S}_{n}$, we compute $\mathrm{U}\left(\mathrm{R}_{n}: \mathrm{S}_{n-1}\right)$ for each value of $\mathrm{S}_{n}$ and then average them together in order to eliminate the effect of $\mathrm{S}_{n}$. Then we have

$$
\mathrm{U}\left(\mathrm{R}_{n}: \mathrm{S}_{n-1} \mid \mathrm{S}_{n}\right)=T_{i}-T_{i j}-T_{i k}+T_{i j k}
$$

which is the information transmission from only $\mathrm{S}_{n-1}$ to $\mathrm{R}_{n}$ and is interpreted as the amount of the effect of $S_{n-1}$ on $R_{n}$ (with the effect of $\mathrm{S}_{n}$ excluded). Similarly, the amount of the effect of $\mathrm{R}_{n-1}$ on $\mathrm{R}_{n}$ with the effects of $\mathrm{S}_{n}$ and $\mathrm{S}_{n-1}$ excluded is

$$
\mathrm{U}\left(\mathrm{R}_{n}: \mathrm{R}_{n-1} \mid \mathrm{S}_{n}, \mathrm{~S}_{n-1}\right)=T_{i k}-T_{i j k}-T_{i k l}+T_{i j k l} \cdot
$$

$\mathrm{U}\left(\mathrm{R}_{n}\right)$ can be expressed as follows:

$$
\begin{aligned}
\mathrm{U}\left(\mathrm{R}_{n}\right)= & \mathrm{U}\left(\mathrm{R}_{n}: \mathrm{S}_{n}\right)+\mathrm{U}\left(\mathrm{R}_{n}: \mathrm{S}_{n-1} \mid \mathrm{S}_{n}\right)+\mathrm{U}\left(\mathrm{R}_{n}: \mathrm{R}_{n-1} \mid \mathrm{S}_{n}, \mathrm{~S}_{n-1}\right) \\
& +\mathrm{U}\left(\mathrm{R}_{n} \mid \mathrm{S}_{n}, \mathrm{~S}_{n-1}, \mathrm{R}_{n-1}\right),
\end{aligned}
$$

where $\mathrm{U}\left(\mathrm{R}_{n} \mid \mathrm{S}_{n}, \mathrm{~S}_{n-1}, \mathrm{R}_{n-1}\right)$ is the residual amount of $\mathrm{U}\left(\mathrm{R}_{n}\right)$ after the effects of $\mathrm{S}_{n}, \mathrm{~S}_{n-1}$, and $\mathrm{R}_{n-1}$ are excluded.

(Manuscript received January 10, 1994; revision accepted for publication March 5, 1995.) 